\title{
DNA methylation in PRDM8 is indicative for dyskeratosis congenita
}

\author{
Carola I. Weidner ${ }^{1,2}$, Qiong Lin ${ }^{1,2}$, Carina Birkhofer ${ }^{3}$, Uwe Gerstenmaier $^{3}$, Andrea \\ Kaifie $^{4}$, Martin Kirschner ${ }^{4}$, Heiko Bruns ${ }^{5}$, Stefan Balabanov 6 , Arne Trummer ${ }^{7}$, \\ Clemens Stockklausner ${ }^{8}$, Britta Höchsmann ${ }^{9,10}$, Hubert Schrezenmeier ${ }^{9,10}$, Marcin \\ Wlodarski ${ }^{11}$, Jens Panse ${ }^{4}$, Tim H. Brümmendorf ${ }^{4}$, Fabian Beier ${ }^{4, *}$ and Wolfgang \\ Wagner ${ }^{1,2, *}$ \\ ${ }^{1}$ Helmholtz-Institute for Biomedical Engineering, RWTH Aachen University Medical Faculty, Aachen, Germany \\ ${ }^{2}$ Institute for Biomedical Technology - Cell Biology, RWTH University Medical School, Aachen, Germany \\ ${ }^{3}$ Varionostic GmbH, Ulm, Germany \\ ${ }^{4}$ Department of Hematology, Oncology, Hemostaseology and Stem Cell Transplantation, RWTH Aachen University Medical \\ Faculty, Aachen, Germany \\ ${ }^{5}$ Department of Internal Medicine 5-Hematology/Oncology, University Hospital Erlangen, Erlangen, Germany \\ ${ }^{6}$ Division of Hematology, University Hospital Zurich, Zurich, Switzerland \\ 7 Department of Hematology, Hemostasis, Oncology, and Stem Cell Transplantation, Hannover Medical School, Hannover, \\ Germany \\ ${ }^{8}$ Department of Pediatric Oncology, Hematology and Immunology, University of Heidelberg, Heidelberg, Germany \\ ${ }^{9}$ Institute of Transfusion Medicine, University of UIm, UIm, Germany \\ 10 Institute of Clinical Transfusion Medicine and Immunogenetics, German Red Cross Blood Transfusion Service Baden- \\ Württemberg-Hessen and University Hospital Ulm, Ulm, Germany \\ ${ }^{11}$ Department of Pediatrics, Hematology and Oncology, University of Freiburg, Freiburg, Germany \\ * These authors have contributed equally to this work \\ Correspondence to: Wolfgang Wagner, email: wwagner@ukaachen.de
}

Fabian Beier, email: fbeier@ukaachen.de

Keywords: aplastic anemia, bone marrow failure, DNA methylation, dyskeratosis congenita, epigenetic, Gerotarget

Received: January 20,2016 Accepted: February 09, $2016 \quad$ Published: February 17, 2016

\section{ABSTRACT}

Dyskeratosis congenita (DKC) is associated with impaired telomere maintenance and with clinical features of premature aging. In this study, we analysed global DNA methylation (DNAm) profiles of DKC patients. Age-associated DNAm changes were not generally accelerated in DKC, but there were significant differences to DNAm patterns of healthy controls, particularly in CpG sites related to an internal promoter region of PR domain containing 8 (PRDM8). Notably, the same genomic region was also hypermethylated in aplastic anemia (AA) - another bone marrow failure syndrome. Site-specific analysis of DNAm level in PRDM8 with pyrosequencing and MassARRAY validated aberrant hypermethylation in 11 DKC patients and 27 AA patients. Telomere length, measured by flow-FISH, did not directly correlate with DNAm in PRDM8. Therefore the two methods may be complementary to also identify patients with still normal telomere length. In conclusion, blood of DKC patients reveals aberrant DNAm patterns, albeit age-associated DNAm patterns are not generally accelerated. Aberrant hypermethylation is particularly observed in PRDM8 and this may support identification and classification of bone marrow failure syndromes. 


\section{INTRODUCTION}

Dyskeratosis congenita (DKC) is a rare disease that is often associated with mutations in genes required for proper telomere maintenance (e.g. DKC1, TERT, RTEL1, TIN2, TERC) [1, 2]. It was the first disease linked to short telomeres [3]. Clinically, DKC usually reflects a triad of oral leukoplakia, nail dystrophy, and skin hyperpigmentation. Other typical manifestations include symptoms of bone marrow failure, and fibrosis of lung and liver [4]. Diagnosis of DKC necessitates awareness in the clinic, but also appropriate laboratory testing of telomere length (TL; usually below the $1 \%$ percentile) and genetic sequencing $[5,6]$. However, not all DKC patients reveal dramatically shortened telomeres [7] and clinically relevant mutations are not always to be found [8]. Acquired aplastic anemia (AA) is another bone marrow failure syndrome that is likewise characterized by telomere attrition $[4,9]$. Correct diagnosis of DKC and AA is important for adequate treatment and more specific biomarkers are urgently wanted [4]. The advances in epigenetic profiling methods provide new perspectives for biomarkers development [10]. Specific DNA methylation (DNAm) changes are indicative for diagnosis, prognosis, choice of therapeutic regimen in many diseases [11]. In this study, we analyzed DNAm profiles of DKC patients to further clarify if age-associated DNAm changes are enhanced in this disease [12] and if aberrant DNAm patterns can be used to support diagnosis of DKC.

\section{RESULTS AND DISCUSSION}

Global DNAm profiles of peripheral blood of four DKC patients were analyzed by Illumina HumanMethylation450 BeadChip technology. We utilized two models for epigenetic age-predictions: a

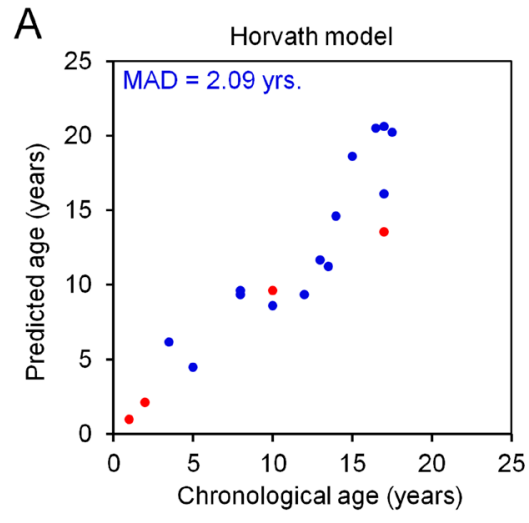

B
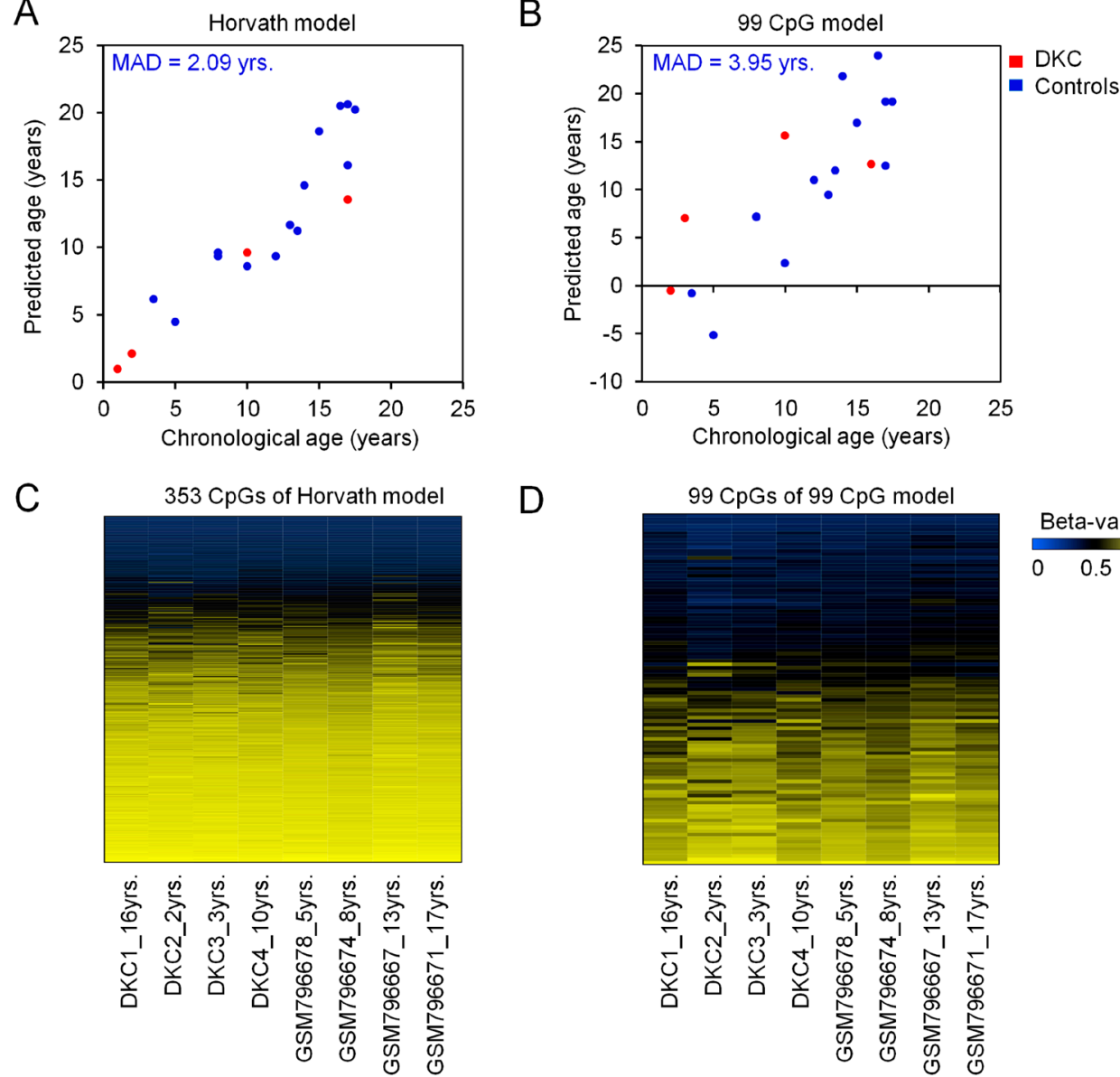

$\mathrm{D}$
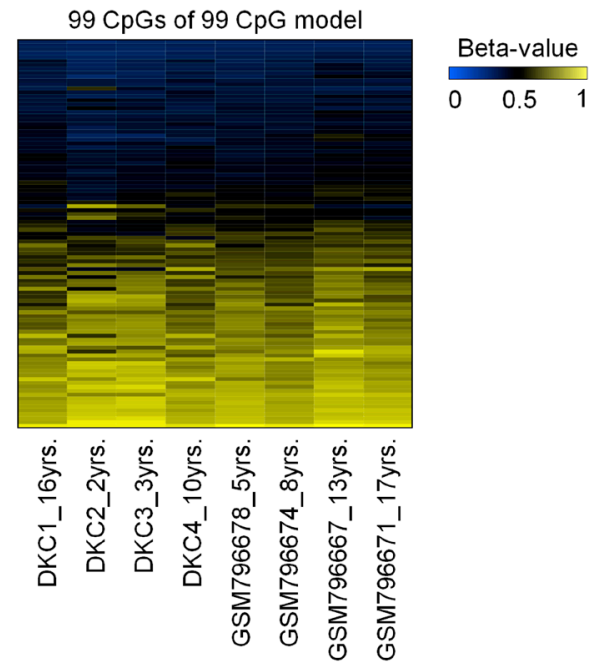

Figure 1: Epigenetic age-predictions of DKC samples. Biological age was estimated in DNAm profiles of four DKC samples (red; whole blood) and healthy control samples (blue; here 14 samples are exemplarily depicted from GSE32148) [33] using a model for epigenetic age-predictions by Horvath A. [13], or our previously published model based on 99 age-associated CpGs B. [12, 14][46]. The mean absolute deviation of predicted and chronological age (MAD) is indicated for the control samples. Heatmaps of the 353 ageassociated CpGs of the Horvath model C. and the 99 age-related CpGs of our age-prediction model D. demonstrate similar DNAm patterns of DKCs patients and controls. Although DKC is considered to be a premature aging syndrome these broader signatures did not reveal the epigenetic age-acceleration in DKC that we previously described for three age-associated CpGs [12]. 
model developed by Horvath that is based on DNAm at 353 age-associated $\mathrm{CpG}$ dinucleotides [13], and our previously published model based on 99 age-associated CpGs [12, 14][]. Overall, the epigenetic age-predictions did not demonstrate general acceleration of age-associated DNAm (Figure 1). This was unexpected, as we previously observed overestimation of biological age using an epigenetic signature based on only three age-associated CpG sites [12]. This discrepancy may partly be attributed to the fact that the linear model for the three-CpGSignature has not been specifically trained for young donors and to the possibility that only specific $\mathrm{CpGs}$ reveal aberrant DNAm in DKC. Genes that are frequently mutated in DKC (such as DKC1, TINF2, TERT, TERC, and $R T E L 1$ ) did not reflect aberrant DNAm patterns (Supplemental Figure 1).

Comparison of DNAm profiles with four age and gender matched controls revealed 764 hypo- and 1,369 hypermethylated CpGs in DKC (adjusted $P$ value $<0.05$; Supplemental Table 1). Hypermethylated CpGs were highly significantly enriched in promoter regions and $\mathrm{CpG}$ islands (Supplemental Figure 2). These results led
A

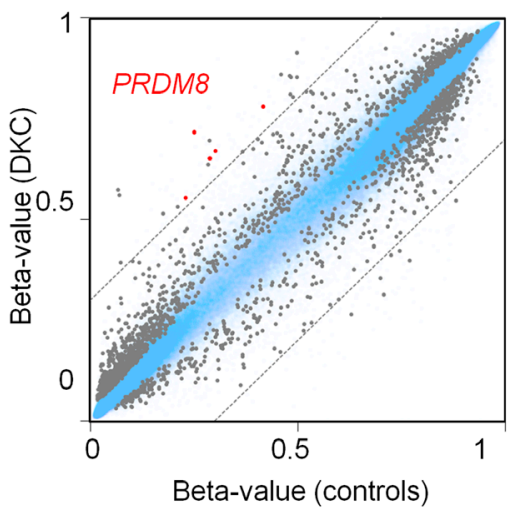

C

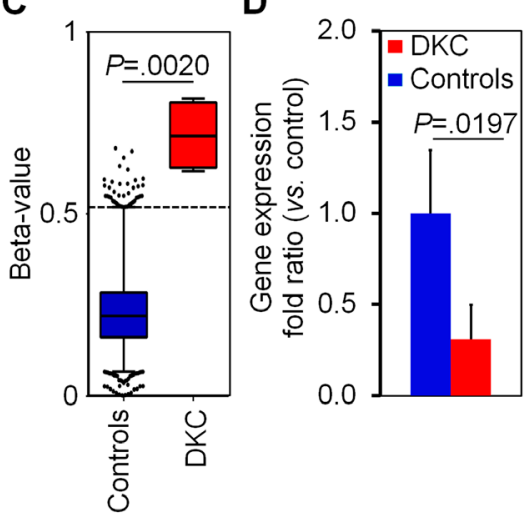

B $\quad P R D M 8$
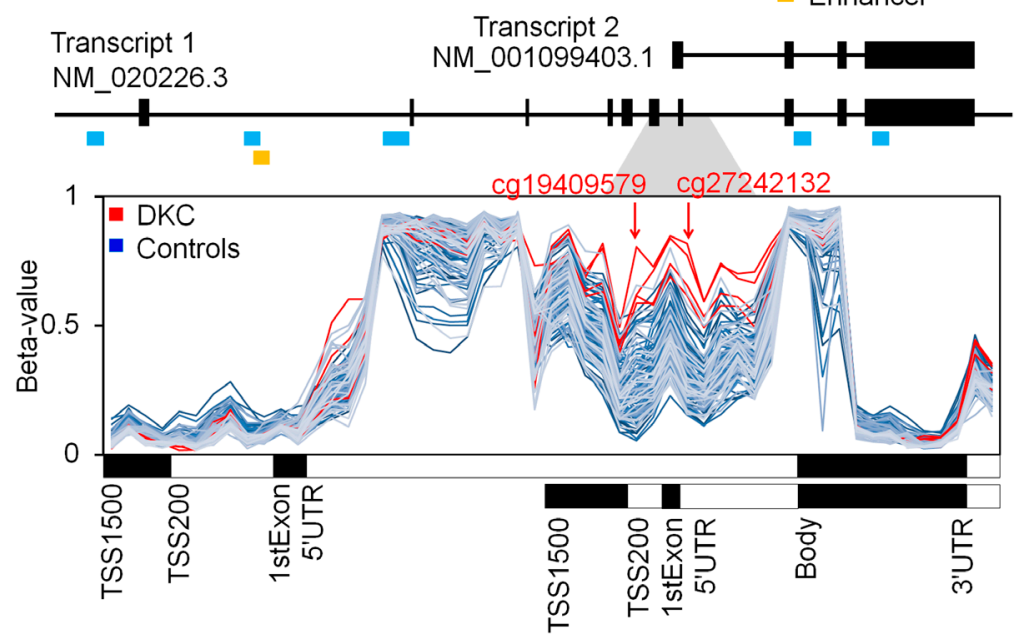

E

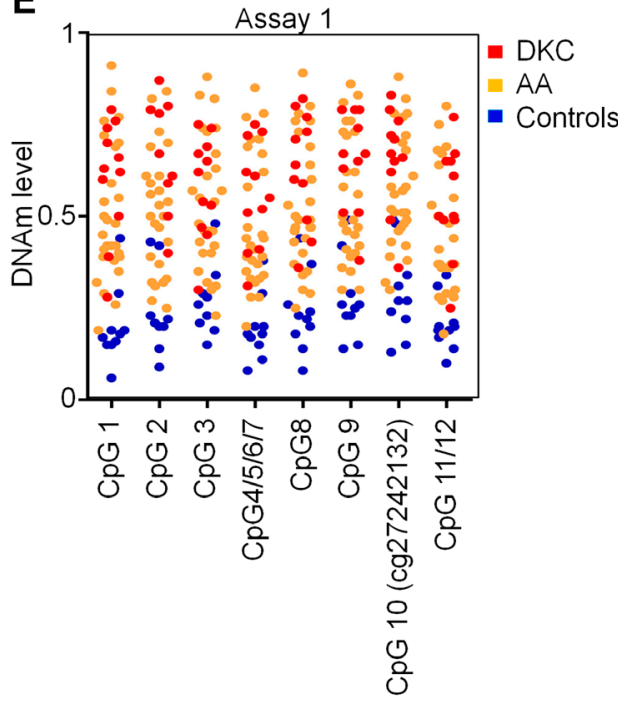

$\mathbf{F}$

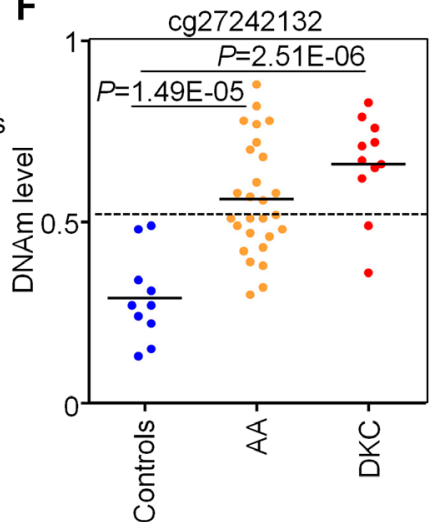

Figure 2: Dyskeratosis congenita is associated with hypermethylation in PRDM8. A. Scatter plots of mean DNAm levels (four DKC patients as compared to four age and gender matched controls) depict 764 hypo- and 1,369 hyper-methylated CpGs in DKC (adjusted $P$ value $<0.05$; relevant CpGs of $P R D M 8$ are indicated in red). B. DNAm levels (beta-values) of CpGs associated with $P R D M 8$ reflect hypermethylation at an internal promoter region in DKC patients (as compared to DNAm profiles of normal blood). The positions of two relevant CpGs are indicated (cg19409579 and cg27242132). C. Boxplots represent distributions of beta-values at cg27242132 in four DKC samples and 4,131 DNAm profiles of blood samples of 16 different studies (see also supplemental Figure 3). The 99 percentile of controls is indicated as dotted line (DNAm level of 52\%). D. Quantitative RT-PCR reveals moderate down-regulation of PRDM8 expression in DKC patients $(n=5$; two-sided Student's $t$-test; $P=0.0197)$. E. MassARRAY analysis of DNAm at cg27242132 $(P R D M 8)$ revealed consistent DNAm levels in neighboring CpGs (controls: $n=10$; AA: $n=27$; DKC: $n=11$ ). F. In comparison to normal controls the DNAm levels at the CpG site cg27242132 were significantly higher in AA $(P=1.49 \mathrm{E}-05)$ and particularly in DKC patients $(P=2.51 \mathrm{E}-06)$. 
us to the assumption, that specific DNAm patterns can be used to support diagnosis of DKC. Therefore, we focused on CpGs with a difference in mean DNAm levels of at least 30\%: $26 \mathrm{CpGs}$ reached this cutoff including five in the gene PR domain containing 8 (PRDM8) and two in PRDM16 (Figure 2A, Supplemental Table 2). It has been demonstrated that PRDM8 is implicated in axon outgrowth $[15,16]$ and regulation of testis steroidogenesis in mice [17] - but hardly anything is known about its potential role in blood formation. In contrast, PRDM16 is involved in human leukemic translocations and was shown to be a physiologic regulator of hematopoietic stem cells [1820]. Notably, PRDM8 and PRDM16 comprise zinc finger motifs as well as PR domains with methyltransferase activity (H3K9) $[16,17]$ making them potential epigenetic modifiers of the histone code.

Hypermethylation in PRDM8 was particularly observed at the CpG sites cg27242132 and cg19409579 corresponding to an internal gene region that was hardly methylated in DNAm profiles of normal blood (analyzed in 4,131 DNAm profiles; Figure $2 \mathrm{~B}$ and 2C). DNAm levels at this region were rather independent from donor age $\left(\mathrm{R}^{2}=0.0311\right.$; Supplemental Figure 3$)$, whereas the cellular composition may be relevant as $P R D M 8$ is slightly higher methylated in lymphocytes than in granulocytes or monocytes (Supplemental Figure 4) [21, 22]. The aberrantly higher DNAm in PRDM8 was also reflected on lower gene expression level of PRDM 8 in qRT-PCR analysis (Figure 2D).

For site-specific analysis of DNAm at PRDM8 we designed pyrosequencing assays for the CpGs cg27242132 (assay 1) and cg19409579 (assay 2). However, the measurements were susceptible for the annealing temperature in PCR (Supplemental Figure 5) and therefore we developed alternative assays based on MassARRAY (Figure 2E and 2F, Supplemental Figure 6). MassARRAY results were very similar to HumanMethylation450
BeadChip data. Analysis of 11 DKC samples demonstrated significantly higher DNAm levels as compared to normal controls $(P=2.51 \mathrm{E}-06$; Figure $2 \mathrm{E}$ and $2 \mathrm{~F})$. Notably, blood samples of 27 AA patients were also hypermethylated at this region $(P=1.49 \mathrm{E}-05)$, suggesting that DNAm in PRDM 8 could serve as a new indicator for bone marrow failure syndromes. As cutoff for normal DNAm level at the CpG site cg27242132 we adopted the 99\% percentile of the 4,131 DNAm profiles of normal blood, corresponding to a DNAm level of 52\% (Figure 2D): Using this cutoff $81.8 \%$ of the DKC (9 of 11 ) and $51.9 \%$ AA patients (14 of 27) were identified as positive.

Subsequently, we correlated DNAm in PRDM8 with telomere length measurements. All DKC patients revealed telomere length below the $1 \%$ percentile in lymphocytes, as measured by flow-FISH, because such telomere attrition has been used as criterion for DKC diagnosis. In contrast, only $7.4 \%$ of AA patients ( 2 of 27 ) were below this threshold (Figure 3 ). Similar results were observed for granulocytes (Supplemental Figure 7). There was no correlation between telomere length and DNAm in PRDM8. Thus, the two biomarkers are independent and can therefore be considered as complementary for diagnosis of both bone marrow syndromes, particularly for those patients that do not reveal significant telomere attrition.

Aberrant hypermethylation in PRDM8 is indicative for DKC and AA, but it is not disease specific. Notably, it was also observed in Down syndrome (DS) - another disease associated with symptoms of premature aging (Supplemental Figure 8) [23]. Analysis in The Cancer Genome Atlas (TCGA) demonstrated that the relevant genomic region was generally higher methylated in tumor tissue (Supplemental Figure 9). We reasoned that DNAm at PRDM8 might be co-regulated with other genomic regions. Using 62 DNAm profiles of acute myeloid leukemia (AML) [24] we identified $151 \mathrm{CpGs}$ showing
A

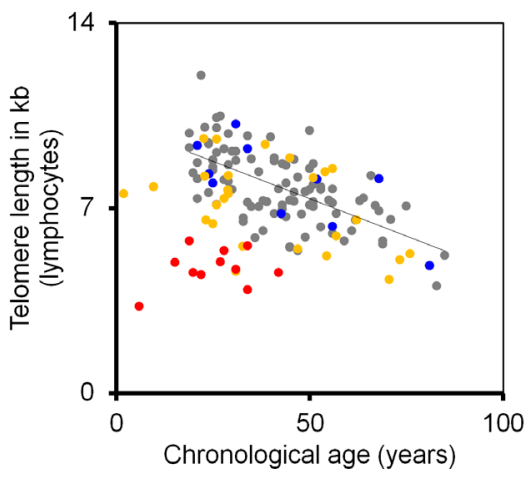

B

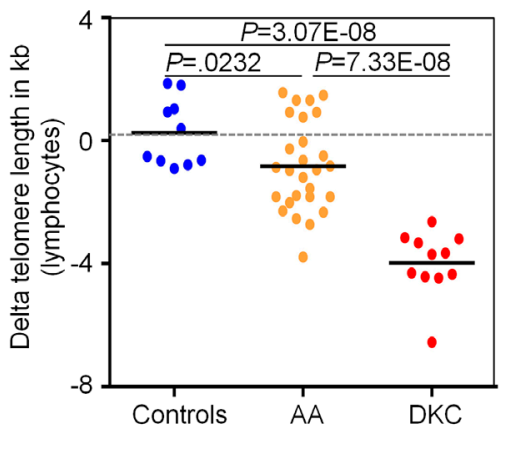

C

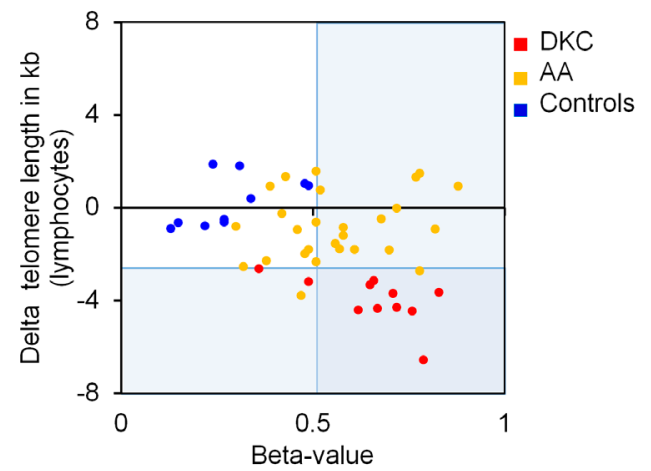

Figure 3: DNAm in PRDM8 is indicative for DKC and AA. A. Telomere length of lymphocytes was measured by flow-FISH for 114 healthy individuals (grey and blue) [12], 11 dyskeratosis congenital patients (DKC; red) and 27 patients with aplastic anemia (AA; yellow). B. Delta telomere length - in relation to age-adjusted mean telomere length in 104 healthy controls - revealed telomere attrition particularly in DKC patients $(P=3.07 \mathrm{E}-08)$, but overall also in AA patients $(P=0.0232)$. C. Delta telomere length was plotted against DNAm in PRDM8 for DKC and AA samples. Cutoffs for 1 percentile in telomere length and 99 percentile in DNAm of $P R D M 8$ are indicated by areas shaded in blue. 
clear correlation with beta-values at cg27242132 (Pearson correlation: $r>0.60$ or $r<-0.60)$. Among these several CpGs corresponded to PRDM16 indicating that the two functionally related genes might also be co-regulated on epigenetic level (Supplemental Figure 10) [25].

\section{CONCLUSIONS}

Age-associated DNAm patterns are not generally accelerated in blood of DKC patients. However, we demonstrate that specific genomic regions reveal aberrant DNAm patterns in this disease. Aberrant hypermethylation was particularly observed at an internal promoter region of PRDM8. Notably, aberrant hypermethylation at the very same genomic region was also observed in patients with AA or Down syndrome - and all of these diseases present clinical features of premature aging. DNAm changes are also reflected on gene expression level and this warrants further functional studies in the future on whether or not PRDM8 directly impacts on cellular aging. On the other hand, we provide simple and cost-effective assays for DNAm measurements in PRDM8 that are even applicable in unsorted peripheral blood. A variety of scoring systems based on TL in different cellular subsets have been proposed to improve diagnosis of AA and DKC [26], but it remains a challenge for patients without severe telomere attrition. DNAm in PRDM8 should be further analyzed in such subsets of patients, in additional cohorts, and other diseases to ultimately demonstrate the clinical potential as a new biomarker.

\section{MATERIALS AND METHODS}

\section{Samples of DKC and AA patients}

Blood samples were obtained from the Registry for Telomeropathies and Aplastic Syndromes of RWTH Aachen University and participating hospitals (15 DKC patients and 32 AA patients). Furthermore, we used blood samples of 14 healthy individuals. The study was approved by the local ethic committee and all samples were taken after written consent (EK206/09). The DKC patients revealed TL below $1 \%$ percentile and diagnosis was complemented by clinical and genotypic characteristics (Supplemental Table 3). Clinical information about AA patients is provided in Supplemental Table 4.

\section{Next-generation sequencing of DKC patients}

The genotype of four patients has already been published before [27-29]. For the other patients we utilized a multiplex-PCR approach (Truseq Amplicon, Illumina $^{\circledR}$ ) if enough DNA was available. To this end, we analyzed 158 amplicons covering 8 telomerase associated genes (NOP10, NHP2, CTC1, DKC1, TERT, TERC, TIN2, RTEL1). 250ng of genomic DNA of 9 DKC samples was prepared according to the TruSeq sample preparation guide. Sequencing data were analyzed using BaseSpace online analysis tool (Illumina, San Diego, CA, USA) and SeqPilot software (JSI medical systems, Ettenheim, Germany). To detect heterozygous or homozygous variants, conditions for scoring a mutant allele were: absolute coverage at SNV-Site $\geq 50$ and relative allele burden of the variant $\geq 30 \%$ of all reads. Further analysis was performed using MutationTaster software (http:// WWW.mutationtaster.org) and PolyPhen-2 software (http://genetics.bwh.harvard.edu/pph2/bgi.shtml) to predict pathogenicity of detected variants.

\section{Telomere length measurement by flow-FISH}

Telomere length was analyzed as described previously $[12,30,31]$. Measurements were performed in triplicates with and without Alexa488-(C3TA2) PNA (Panagene, South Korea). Cow thymocytes were used as an internal control to calculate telomere length in kilo bases $(\mathrm{kb})$. Identification of lymphocytes, granulocytes and cow thymocytes was done based on forward scatter properties and LDS 751 fluorescence. Peripheral blood of 104 healthy controls was used as for age-adaption as described previously $[12,30]$.

\section{DNA methylation profiles}

Genomic DNA was isolated from four DKC patients with the QIAamp DNA Blood Midi Kit (Qiagen, Hilden, Germany; these samples are indicated in Supplemental Table 3). DNA quality was assessed with a NanoDrop ND-1000 spectrometer and by gel electrophoresis and then analyzed with the Illumina HumanMethylation450 BeadChip (Illumina). This platform can assay more than 480,000 $\mathrm{CpG}$ sites at single base resolution (covering $99 \%$ of RefSeq genes and $96 \%$ of CpG islands) [32]. Hybridization and initial data analysis using the BeadStudio Methylation module was performed at the DKFZ Gene Core Facility (Heidelberg, Germany). Raw data are available at Gene Expression Omnibus under the accession number GSE75310. Beta-values ranging from 0 (non-methylated) to 1 (100\% methylation) are provided for each $\mathrm{CpG}$ site.

In addition, we utilized the following publically available datasets of normal peripheral blood: GSE32148 [33], GSE30870 [34], GSE36064 [35], GSE40005, GSE40279 [36], GSE41169 [37], GSE42861 [38], GSE50660 [39], GSE51180 [40], GSE51388, GSE56046 [41], GSE56105 [42], GSE56581 [41], GSE58651 [43], GSE61496 [44], GSE62992, and GSE49064 [45]. DNAm profiles of patients with Down syndrom were 
retrieved from GSE52588 [23]. AML profiles were used from a dataset by Qu et al. (GSE58477) [24]. Additional DNAm values were downloaded from the UCSC Cancer Genomics Browser (www.genome-cancer.ucsc.edu/) for various types of cancer as indicated. Unpaired limma $t$-test was calculated in $\mathrm{R}$ to select for significantly changed CpG sites (adjusted $P$ value $<0.05$ ).

\section{Pyrosequencing of PRDM8}

Site-specific analysis of DNAm levels in PRDM8 was analyzed for 14 controls, 13 DKC, and 20 AA samples. Primers were designed for two different regions (assay 1 and assay 2). $100 \mathrm{ng}$ of DNA was bisulfite converted using the EZ DNA Methylation Kit (Zymo, Irvine, CA, USA) and amplified by PCR with annealing temperatures of $56^{\circ} \mathrm{C}$ and $65^{\circ} \mathrm{C}$, respectively. After amplification $20 \mu \mathrm{l}$ of the PCR product was immobilized to $5 \mu \mathrm{l}$ of Streptavidin beads (GE Healthcare, Piscataway, NJ, USA) and annealed to $0.8 \mu \mathrm{l}$ sequencing primer $(20 \mu \mathrm{M})$ for 2 min at $80^{\circ} \mathrm{C}$. For pyrosequencing the PyorMark Q96 ID system was utilized and analysis was performed with the PyroMark Q CpG software (Qiagen, Hilden, Germany). Primers: assay 1 forward: 5'-biotin-GGGGTTGTTT ATTGTTAGTA ATATTGTATA AAAGGAGGA-3'; assay 1 reverse: 5'-ACCCCGCTCT AAACCCAAAT TCTT-3'; assay 1 sequencing: 5'-GCCTACCCTA AAAATATACC-3'; assay 2 forward: 5'-AGTGAAACGG GGAAAGGTTT TTTTTAATTA TTTTGG-3'; assay 2 reverse: 5'-biotin-GTCCCCTCCC TTTAACTCTT TACTAAACCAA-3'; assay 2 sequencing 5'-TTTTGAGAGG CGTTGTTATT-3'.

\section{MassArray of PRDM8}

Alternatively, we used MassARRAY for sitespecific analysis of DNAm levels. This highly sensitive method is based on MALDI-TOF mass spectrometry and measurements were performed at Varionostic $\mathrm{GmbH}$ (www.varionostic.de; Ulm, Germany). Samples of 10 healthy controls, $11 \mathrm{DKC}$, and $27 \mathrm{AA}$ patients were analyzed. Amplicons were designed with the Sequenom's EpiDESIGNER software. $150 \mathrm{ng}$ of DNA was bisulfite converted with the EZ DNA Methylation Gold Kit (Zymo). Converted DNA was amplified by PCR using the HotStart Plus PCR Master Mix (Qiagen). Unincorporated dNTPs were neutralized using shrimp alkaline phosphatase (Agena Bioscience, San Diego, CA, USA). Subsequently, $10 \mu \mathrm{l}$ of PCR product was in vitro transcribed and cleaved in a base-specific (U-specific) manner using RNase A (T-Cleavage MassCleave Kit; Agena Bioscience). The cleaved products were then analyzed by the MALDITOF mass spectrometer. Mass differences of $16 \mathrm{Da}$ or $\mathrm{n}$-fold were representative for methylation events. The experiment was performed with a MassARRAY
Analyzer 4 System (Agena Bioscience). Primers: assay 1 forward: 5'-aggaagagag TTTTTGAGGG GTTGTTTATT GTTAGT-3'; assay 1 reverse: 5'-cagtaatacg actcactata gggagaaggc tTACCCTAAAAATATACCCC AAAACC-3'; assay 2 forward: 5'-aggaagagag GGGGAAAGGT TTTTTTTAAT TATTTT-3'; assay 2 reverse: $5^{\prime}$-cagtaatacg actcactata gggagaagge tCCCTCCCTT TAACTCTTTA CTAAACC-3' (Bases that correspond to the converted sequence of PRDM8 are indicated in capital letters).

\section{qRT-PCR of PRDM8}

Expression of PRDM8 was analyzed by real-time quantitative PCR (RT-qPCR) using the StepOneTM Instrument (Applied Biosystems, Applera Deutschland $\mathrm{GmbH}$, Darmstadt, Germany). 250ng RNA was reversetranscribed using the high capacity cDNA Reverse Transcription Kit (Applied Biosystems). cDNA was amplified using Power SYBR Green PCR Master Mix (Applied Biosystems). Gene expression was normalized to $G A P D H$. The following primers (Metabion, Martinsried, Germany) were used for amplification of PRDM8: forward: 5'-ACCAGCGTTT ACACCACCTG-3'; reverse: 5'-CCATTTGCTG CTGAGGTGTC-3'.

\section{ACKNOWLEDGMENTS}

We thank Lucia Vankann for excellent technical assistance. List of Funding Sources: This work was supported by the Else Kröner-Fresenius Stiftung (2014 A193;W.W.), by the German Research Foundation (WA/1706/2-1;W.W.), by the Interdisciplinary Center for Clinical Research (IZKF) in the Faculty of Medicine at the RWTH Aachen University (O1-1;W.W.), and partly by a grant from Lichterzellen e.V. (T.H.B.). Author Contribution: C.I.W., F.B., and W.W. designed research, analyzed and interpreted data; C.I.W., F.B., Q.L., C.B., U.G., A.K., and M.K. performed experiments; H.B., S.B., A.T., C.S., B.H., H.S., M.W., J.P., and T.H.B. contributed vital reagents; C.I.W. F.B. and W.W. wrote the manuscript, and all authors provided input to the manuscript.

\section{CONFLICTS OF INTERESTS}

RWTH Aachen University has applied for a patent application on DNAm in PRDM8 (DE 102015121 969.7) and W.W. and U.G. are involved in the company Cygenia (wWw.cygenia.com) that might provide service to this method. The remaining authors declare no competing financial interests.

\section{REFERENCES}

1. Blasco M. A. Telomeres and human disease: ageing, cancer and beyond. Nat Rev Genet. 2005; 6: 611-622. 
2. Young N. S. Telomere biology and telomere diseases: implications for practice and research. Hematology Am Soc Hematol Educ Program. 2010; 2010: 30-35.

3. Vulliamy T., Marrone A., Goldman F., Dearlove A., Bessler M., Mason P. J., Dokal I. The RNA component of telomerase is mutated in autosomal dominant dyskeratosis congenita. Nature. 2001; 413: 432-435.

4. Calado R. T., Young N. S. Telomere diseases. N Engl J Med. 2009; 361: 2353-2365.

5. Townsley D. M., Dumitriu B., Young N. S. Bone marrow failure and the telomeropathies. Blood. 2014; 124: 27752783.

6. Alter B. P., Baerlocher G. M., Savage S. A., Chanock S. J., Weksler B. B., Willner J. P., Peters J. A., Giri N., Lansdorp P. M. Very short telomere length by flow fluorescence in situ hybridization identifies patients with dyskeratosis congenita. Blood. 2007; 110: 1439-1447.

7. Aubert G., Baerlocher G. M., Vulto I., Poon S. S., Lansdorp P. M. Collapse of telomere homeostasis in hematopoietic cells caused by heterozygous mutations in telomerase genes. PLoS Genet. 2012; 8: e1002696.

8. Dokal I., Vulliamy T., Mason P., Bessler M. Clinical utility gene card for: Dyskeratosis congenita - update. 2015. Eur J Hum Genet. 2015; 23: 1-4.

9. Brummendorf T. H., Maciejewski J. P., Mak J., Young N. S., Lansdorp P. M. Telomere length in leukocyte subpopulations of patients with aplastic anemia. Blood. 2001; 97: 895-900.

10. Bock C. Epigenetic biomarker development. Epigenomics. 2009; 1: 99-110.

11. Herceg Z., Hainaut P. Genetic and epigenetic alterations as biomarkers for cancer detection, diagnosis and prognosis. Mol Oncol. 2007; 1: 26-41.

12. Weidner C. I., Lin Q., Koch C. M., Eisele L., Beier F., Ziegler P., Bauerschlag D. O., Jockel K. H., Erbel R., Muhleisen T. W., Zenke M., Brummendorf T. H., Wagner W. Aging of blood can be tracked by DNA methylation changes at just three CpG sites. Genome Biol. 2014; 15: R24.

13. Horvath S. DNA methylation age of human tissues and cell types. Genome Biol. 2013; 14: R115.

14. Lin Q., Wagner W. Epigenetic Aging Signatures Are Coherently Modified in Cancer. PLoS Genet. 2015; 11: e1005334.

15. Ross S. E., McCord A. E., Jung C., Atan D., Mok S. I., Hemberg M., Kim T. K., Salogiannis J., Hu L., Cohen S., Lin Y., Harrar D., McInnes R. R., et al. Bhlhb5 and Prdm8 form a repressor complex involved in neuronal circuit assembly. Neuron. 2012; 73: 292-303.

16. Hohenauer T., Moore A. W. The Prdm family: expanding roles in stem cells and development. Development. 2012; 139: 2267-2282.

17. Eom G. H., Kim K., Kim S. M., Kee H. J., Kim J. Y., Jin H. M., Kim J. R., Kim J. H., Choe N., Kim K. B., Lee J.,
Kook H., Kim N., et al. Histone methyltransferase PRDM8 regulates mouse testis steroidogenesis. Biochem Biophys Res Commun. 2009; 388: 131-136.

18. Aguilo F., Avagyan S., Labar A., Sevilla A., Lee D. F., Kumar P., Lemischka I. R., Zhou B. Y., Snoeck H. W. Prdm16 is a physiologic regulator of hematopoietic stem cells. Blood. 2011; 117: 5057-5066.

19. Chuikov S., Levi B. P., Smith M. L., Morrison S. J. Prdm16 promotes stem cell maintenance in multiple tissues, partly by regulating oxidative stress. Nat Cell Biol. 2010; 12: 9991006.

20. Deneault E., Cellot S., Faubert A., Laverdure J. P., Frechette M., Chagraoui J., Mayotte N., Sauvageau M., Ting S. B., Sauvageau G. A functional screen to identify novel effectors of hematopoietic stem cell activity. Cell. 2009; 137: 369-379.

21. Reinius L. E., Acevedo N., Joerink M., Pershagen G., Dahlen S. E., Greco D., Soderhall C., Scheynius A., Kere J. Differential DNA methylation in purified human blood cells: implications for cell lineage and studies on disease susceptibility. PLoS ONE. 2012; 7: e41361.

22. Zilbauer M., Rayner T. F., Clark C., Coffey A. J., Joyce C. J., Palta P., Palotie A., Lyons P. A., Smith K. G. Genomewide methylation analyses of primary human leukocyte subsets identifies functionally important cell-type-specific hypomethylated regions. Blood. 2013; 122: e52-e60.

23. Bacalini M. G., Gentilini D., Boattini A., Giampieri E., Pirazzini C., Giuliani C., Fontanesi E., Scurti M., Remondini D., Capri M., Cocchi G., Ghezzo A., Del R. A., et al. Identification of a DNA methylation signature in blood cells from persons with Down Syndrome. Aging (Albany NY). 2015; 7: 82-96.

24. Qu Y., Lennartsson A., Gaidzik V. I., Deneberg S., Karimi M., Bengtzen S., Hoglund M., Bullinger L., Dohner K., Lehmann S. Differential methylation in CN-AML preferentially targets non-CGI regions and is dictated by DNMT3A mutational status and associated with predominant hypomethylation of HOX genes. Epigenetics. 2014; 9: 1108-1119.

25. Fumasoni I., Meani N., Rambaldi D., Scafetta G., Alcalay M., Ciccarelli F. D. Family expansion and gene rearrangements contributed to the functional specialization of PRDM genes in vertebrates. BMC Evol Biol. 2007; 7: 187.

26. Alter B. P., Rosenberg P. S., Giri N., Baerlocher G. M., Lansdorp P. M., Savage S. A. Telomere length is associated with disease severity and declines with age in dyskeratosis congenita. Haematologica. 2012; 97: 353-359.

27. Stockklausner C., Raffel S., Klermund J., Bandapalli O. R., Beier F., Brummendorf T. H., Burger F., Sauer S. W., Hoffmann G. F., Lorenz H., Tagliaferri L., Nowak D., Hofmann W. K., et al. A novel autosomal recessive TERT T1129P mutation in a dyskeratosis congenita family leads to cellular senescence and loss of CD34+ hematopoietic stem cells not reversible by mTOR-inhibition. Aging 
(Albany NY). 2015; 7: 911-927.

28. Trautmann K., Jakob C., von G. U., Schleyer E., Brummendorf T. H., Siegert G., Ehninger G., Platzbecker U. Eltrombopag fails to improve severe thrombocytopenia in late-stage dyskeratosis congenita and diamond-blackfananaemia. Thromb Haemost. 2012; 108: 397-398.

29. Stepensky P., Rensing-Ehl A., Gather R., Revel-Vilk S., Fischer U., Nabhani S., Beier F., Brummendorf T. H., Fuchs S., Zenke S., Firat E., Pessach V. M., Borkhardt A., et al. Early-onset Evans syndrome, immunodeficiency, and premature immunosenescence associated with tripeptidylpeptidase II deficiency. Blood. 2015; 125: 753-761.

30. Beier F., Masouleh B. K., Buesche G., Ventura Ferreira M. S., Schneider R. K., Ziegler P., Wilop S., Vankann L., Gattermann N., Platzbecker U., Giagounidis A., Gotze K. S., Nolte F., et al. Telomere dynamics in patients with del (5q) MDS before and under treatment with lenalidomide. Leuk Res. 2015. Sep 21. pii: S0145-2126(15)30380-5

31. Werner B., Beier F., Hummel S., Balabanov S., Lassay L., Orlikowsky T., Dingli D., Brummendorf T. H., Traulsen A. Reconstructing the dynamics of hematopoietic stem cells from telomere length distributions. Elife. 2015; 4.

32. Bibikova M., Barnes B., Tsan C., Ho V., Klotzle B., Le J. M., Delano D., Zhang L., Schroth G. P., Gunderson K. L., Fan J. B., Shen R. High density DNA methylation array with single CpG site resolution. Genomics. 2011; 98: 288295.

33. Harris R. A., Nagy-Szakal D., Pedersen N., Opekun A., Bronsky J., Munkholm P., Jespersgaard C., Andersen P., Melegh B., Ferry G., Jess T., Kellermayer R. Genome-wide peripheral blood leukocyte DNA methylation microarrays identified a single association with inflammatory bowel diseases. Inflamm Bowel Dis. 2012; 18: 2334-2341.

34. Heyn H., Li N., Ferreira H. J., Moran S., Pisano D. G., Gomez A., Diez J., Sanchez-Mut J. V., Setien F., Carmona F. J., Puca A. A., Sayols S., Pujana M. A., et al. Distinct DNA methylomes of newborns and centenarians. Proc Natl Acad Sci U S A. 2012; 109: 10522-10527.

35. Alisch R. S., Barwick B. G., Chopra P., Myrick L. K., Satten G. A., Conneely K. N., Warren S. T. Age-associated DNA methylation in pediatric populations. Genome Res. 2012; 22: 623-632.

36. Hannum G., Guinney J., Zhao L., Zhang L., Hughes G., Sadda S., Klotzle B., Bibikova M., Fan J. B., Gao Y., Deconde R., Chen M., Rajapakse I., et al. Genome-wide Methylation Profiles Reveal Quantitative Views of Human Aging Rates. Mol Cell. 2013; 49: 459-367.

37. Horvath S., Zhang Y., Langfelder P., Kahn R. S., Boks M. P., van E. K., van den Berg L. H., Ophoff R. A. Aging effects on DNA methylation modules in human brain and blood tissue. Genome Biol. 2012; 13: R97.

38. Liu Y., Aryee M. J., Padyukov L., Fallin M. D., Hesselberg E., Runarsson A., Reinius L., Acevedo N., Taub M., Ronninger M., Shchetynsky K., Scheynius A., Kere J., et al. Epigenome-wide association data implicate DNA methylation as an intermediary of genetic risk in rheumatoid arthritis. Nat Biotechnol. 2013; 31: 142-147.

39. Tsaprouni L. G., Yang T. P., Bell J., Dick K. J., Kanoni S., Nisbet J., Vinuela A., Grundberg E., Nelson C. P., Meduri E., Buil A., Cambien F., Hengstenberg C., et al. Cigarette smoking reduces DNA methylation levels at multiple genomic loci but the effect is partially reversible upon cessation. Epigenetics. 2014; 9: 1382-1396.

40. Cruickshank M. N., Oshlack A., Theda C., Davis P. G., Martino D., Sheehan P., Dai Y., Saffery R., Doyle L. W., Craig J. M. Analysis of epigenetic changes in survivors of preterm birth reveals the effect of gestational age and evidence for a long term legacy. Genome Med. 2013; 5: 96.

41. Reynolds L. M., Taylor J. R., Ding J., Lohman K., Johnson C., Siscovick D., Burke G., Post W., Shea S., Jacobs D. R., Jr., Stunnenberg H., Kritchevsky S. B., Hoeschele I., et al. Age-related variations in the methylome associated with gene expression in human monocytes and T cells. Nat Commun. 2014; 5: 5366.

42. McRae A. F., Powell J. E., Henders A. K., Bowdler L., Hemani G., Shah S., Painter J. N., Martin N. G., Visscher P. M., Montgomery G. W. Contribution of genetic variation to transgenerational inheritance of DNA methylation. Genome Biol. 2014; 15: R73.

43. Dhas D. B., Ashmi A. H., Bhat B. V., Kalaivani S., Parija S. C. Comparison of genomic DNA methylation pattern among septic and non-septic newborns - An epigenome wide association study. Genom Data. 2015; 3: 36-40.

44. Tan Q., Frost M., Heijmans B. T., von Bornemann H. J., Tobi E. W., Christensen K., Christiansen L. Epigenetic signature of birth weight discordance in adult twins. BMC Genomics. 2014; 15: 1062.

45. Steegenga W. T., Boekschoten M. V., Lute C., Hooiveld G. J., de Groot P. J., Morris T. J., Teschendorff A. E., Butcher L. M., Beck S., Muller M. Genome-wide age-related changes in DNA methylation and gene expression in human PBMCs. Age (Dordr ). 2014; 36: 9648.

46. Lin Q., Weidner C.I., Costa I., Marioni R., Ferreira M., Deary I. and Wagner W. DNA methylation levels at individual age-associated $\mathrm{CpG}$ sites can be indicative for life expectancy. Aging (Albany NY). 2016; 8:394-401. 